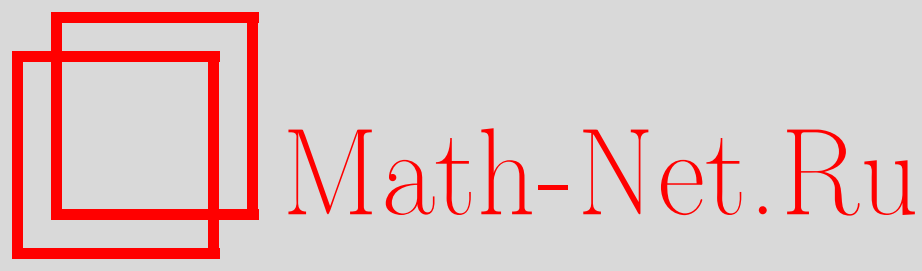

М. Джанфреда, Д. Ландольфи, Неавтономные гамильтоновы квантовые системы, операторные уравнения и представления упорядоченного по Вейлю базиса Бендера-Данна при зависящих от времени канонических преобразованиях, ТМФ, 2017, том 193, номер 1, 4165

DOI: https://doi.org/10.4213/tmf9256

Использование Общероссийского математического портала Math-Net.Ru подразумевает, что вы прочитали и согласны с пользовательским соглашением http://www . mathnet.ru/rus/agreement

Параметры загрузки:

IP : 54.80 .97 .219

26 апреля 2023 г., 12:22:22

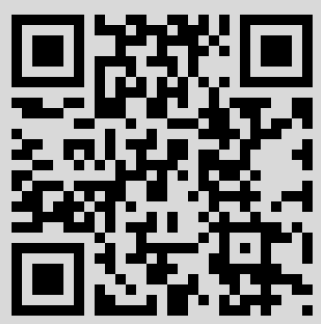




\title{
НЕАВТОНОМНЫЕ ГАМИЛЬТОНОВЫ КВАНТОВЫЕ СИСТЕМЫ, ОПЕРАТОРНЫЕ УРАВНЕНИЯ И ПРЕДСТАВЛЕНИЯ УПОРЯДОЧЕННОГО ПО ВЕЙЛЮ БАЗИСА БЕНДЕРА-ДАННА ПРИ ЗАВИСЯЩИХ ОТ ВРЕМЕНИ КАНОНИЧЕСКИХ ПРЕОБРАЗОВАНИЯХ
}

\begin{abstract}
С использованием зависящих от времени канонических преобразований решена проблема интегрирования операторных уравнений, описывающих динамику неавтономных квантовых систем. Исследуемые операторные уравнения в сущности воспроизводят на квантовом уровне классические условия интегрируемости для основных случаев одномерных неавтономных динамических систем. Решения ищутся в виде операторных рядов в базисе псевдодифференциальных операторов Бендера-Данна. Наряду с данной проблемой рассмотрены квантовые канонические преобразования. Минимальное решение операторного уравнения в представлении данного базиса при фиксированном времени соответствует наименьшему порядку вклада от решения, полученного в результате применения канонического линейного преобразования к элементам базиса.
\end{abstract}

Ключевые слова: упорядоченность по Вейлю, операторный базис Бендера-Данна, операторные уравнения, зависящие от времени квантовые системы, квантовые канонические преобразования.

DOI: https://doi.org/10.4213/tmf9256

Работа М. Джанфреды поддержана Japan Society for Promotion of Science (грант PE14011).

*Institute of Industrial Science, University of Tokyo, Tokyo, Japan.

E-mail: mariagiovanna.gianfreda@gmail.com

†Museo Storico della Fisica e Centro Studi e Ricerche "Enrico Fermi", Roma, Italy

${ }^{\ddagger}$ IFAC-CNR, Istituto di Fisica Applicata "Nello Carrara", Consiglio Nazionale delle Ricerche, Sesto Fiorentino, Italy

${ }^{\S}$ Dipartimento di Matematica e Fisica "Ennio De Giorgi", Universitá del Salento and I.N.F.N. Sezione di Lecce, Lecce, Italy. E-mail: giulio.landolf@@unisalento.it, giulio.landolfi@le.infn.it 


\section{1. ВВЕДЕНИЕ}

Целью настоящей статьи является исследование возможных преимуществ и трудностей, возникающих при изучении зависящих от времени операторных уравнений, описывающих неавтономные квантовые гамильтоновы системы, таких как уравнения для операторов типа действие-угол, с помощью зависящих от времени канонических преобразований. Мотивирующим фактором служит то обстоятельство, что к операторным уравнениям в фазовом пространстве обычно обращаются в контексте консервативных систем и при этом рассматривают операторный базис в представлении Шредингера, учитывающем подходящее упорядочивание (см., например, статьи [1]-[6]). Следовательно, можно указать две причины провести данное исследование.

С одной стороны, мы заинтересованы в решении конкретных примеров операторных уравнений в фазовом пространстве, свойственных неавтономным системам. С учетом этого мы исследуем условия интегрируемости (в смысле теоремы Лиувилля) одномерных неавтономных систем, переходя на квантовый уровень. Поводом для рассмотрения данной конкретной проблемы является наше намерение использовать некоторые базовые свойства, присущие формализму на основе симметрии, поскольку это может привести к более точному описанию общих положений классической и квантовой механики и улучшению понимания квазиклассических режимов и переходов из классической механики в квантовую (например, хаотических динамик) и наоборот. В свете сказанного представляется разумным обратить внимание на независимые сохраняющиеся величины и алгебры, лежащие в основе обозначенной проблемы и являющиеся ключевыми составляющими базирующихся на соображениях симметрии подходов. Помимо того что условия типа действие-угол представляют собой содержательный класс уравнений с точки зрения теории динамических систем, они также являются простейшим примером операторных уравнений в алгебраическом смысле. В самом деле, в случае автономных гамильтоновых систем определяющее задачу уравнение отвечает условию, согласно которому искомый оператор формально сопряжен с гамильтонианом $H(q, p)$. Исследования по этому поводу можно найти, например, в статьях [1], [2] или в работе [7], где рассматривается ограниченное координатное пространство. В случае неавтономных систем гамильтониан не является производящей функцией (сохраняющимся зарядом) для трансляций времени и условие интегрируемости переписывается в терминах динамических переменных действие-угол, формально сопряженных с инвариантом действия $I(q, p, t)$. Очевидно, что в случае неавтономных систем рассматриваемая проблема имеет некоторые общие черты технического плана со случаем автономных гамильтоновых систем, но является, безусловно, более сложной. Кроме того, ей присущи аналогичные концептуальные особенности, такие как, например, неединственность реализации решений операторных уравнений.

Второй интересующий нас вопрос - какое влияние оказывают канонические преобразования на квантовом уровне. Мы рассматриваем эту проблему, дополняя обозначенное в предыдущем абзаце исследование. А именно, мы решаем уравнения, определяющие операторы угла, сопряженные с заданными инвариантами, в некоторых простых случаях неавтономных гамильтонианов, попутно сравнивая подходы к решению этих уравнений, основанные на использовании двух различных опе- 
раторных базисов для пространства решений, которые связаны между собой каноническим преобразованием. Такой подход позволяет упростить использование отображения на множество минималъных решений (терминология заимствована из статьи [1]). Два базиса, которые мы намереваемся использовать, суть упорядоченный по Вейлю базис Бендера-Данна [1] и базис, получаемый посредством применения к базису Бендера-Данна зависящего от времени канонического преобразования, приводящего к заданию инварианта. Следовательно, можно считать, что обозначенное выше сравнение проясняет связь между результатами для упорядоченных по Вейлю операторных базисов типа базиса Шредингера и Гейзенберга. После осуществления канонических отображений мы стремимся вывести точные формулы, выражающие элементы одного базиса через элементы другого. Что касается канонических отображений, то мы используем линейные зависящие от времени канонические преобразования для координат и импульса. Мы рассматриваем данные отображения в фазовом пространстве в силу их простоты, а также следуя направлению исследования, предпринятого Мустафазаде в статье [8], и подходу, согласно которому симплектические методы перемасштабирования времени привлекаются для разработки адаптивных методов вычислений в молекулярной динамике [9]. Более того, известно, что подобная схема возникает при канонических преобразованиях, превращающих зависящие от времени квадратичные системы в не зависящие от времени (см., например, работу [10] и ссылки в ней), так что некоторые упомянутые результаты могут оказаться полезными при решении поставленных в настоящей статье задач и проверке полученных результатов.

Основываясь на сказанном выше, мы обсуждаем основные примеры одномерных неавтономных динамических систем, пользуясь тем, что их гамильтонианы можно привести посредством зависящих от времени линейных канонических преобразований в автономную форму с точностью до зависящего от времени множителя. Это применимое на практике свойство позволяет без труда облегчить интегрирование операторного уравнения при сохранении его неавтономной формы. Также оно дает возможность формализовать и исследовать некоторые простые, абсолютно хрестоматийные неавтономные случаи, такие как случаи линейных, квадратичных потенциалов и потенциалов четвертого порядка. В результате для них мы подробно выводим производящие функции для коэффициентов, фигурирующих в минимальном представлении рядов решений операторных уравнений типа действие-угол. Мы получаем в явном виде операторы, которые осуществляют произвольные линейные преобразования базиса Бендера-Данна, выражая их в терминах исходных упорядоченных по Вейлю базисных элементов, и, наконец, переходим к тщательному изучению влияния квантовых линейных канонических преобразований на формальную структуру решений исследуемых операторных уравнений.

Содержание настоящей статьи имеет следующий вид. В разделе 2 введены необходимые нам зависящие от времени линейные преобразования фазового пространства, а также рассмотрен сопутствующий им унитарный оператор с целью продемонстрировать, что если соответствующий анзац остается верным, то преобразования могут превратить неавтономную систему в эквивалентную ей автономную. В разделе 3 рассмотрено действие этих преобразований на операторный базис Бендера-Данна (12). Мы имеем дело в том числе с отрицательными степенями операторов, а полученные формулы дополняют результаты статьи [11]. Раздел 4 посвящен вы- 
работке непосредственного понимания на уровне интуиции структурной разницы между формальными координатно-импульсными разложениями квантованных по Вейлю классических решений условия интегрируемости для одномерных гамильтоновых систем и разложениями квантовых решений (включая минимальные). В разделах 5-7 мы детально исследуем условие интегрируемости для неавтономных одномерных квантовых систем, подвергающихся воздействию линейного, квадратичного потенциала или потенциала четвертого порядка. Раздел 8 посвящен краткому повторению и заключительному обсуждению результатов. Наконец, в приложении вкратце рассмотрен метод обратной задачи Лиувилля.

\section{2. ЛИНЕЙНЫЕ ПРЕОБРАЗОВАНИЯ ФАЗОВОГО ПРОСТРАНСТВА}

Изучение неавтономных систем с гамильтонианом $H_{\text {na }}(p, q, t)$ можно увязать в конечном счете с изучением контактных преобразований в расширенном фазовом пространстве, поскольку получающийся в результате преобразований гамильтониан оказывается на самом деле автономным. На практике это означает, что мы используем расширенные канонические преобразования $(q, p, t) \rightarrow(Q, P, \tau)$, перемешивающие пространственные переменные и импульсы (координаты или операторы, зависящие от динамического режима) посредством зависящего от времени отображения фазового пространства, переопределяющего переменную времени (см., например, статью [12]).

Как мы уже сказали, основная пара $Q, P$, появляющаяся при исследовании интересующих нас неавтономных гамильтонианов, линейна по исходной канонической паре переменных $(q, p)$. Чтобы пояснить, зачем мы ее используем, рассмотрим зависящий от времени квантовый гамильтониан с одной степенью свободы

$$
H=\frac{p^{2}}{2 m(t)}+V(q, t) .
$$

В общем случае унитарное преобразование $U(q, p, t)$ превращает исходный гамильтониан (1) в новый оператор $H^{\prime}$ согласно формуле

$$
H^{\prime}=U^{-1} H U-i U^{-1} \partial_{t} U
$$

Можно попробовать преобразовать гамильтониан (1) в новый гамильтониан, имеющий удобную форму. Рассмотрим, в частности, расширенные канонические преобразования, линейные по пространственным переменным и импульсам, определенные выражениями

$$
Q=A(t) q, \quad P=B(t) p+C(t) q, \quad \text { где } \quad A(t) B(t)=1 .
$$

Следует отметить, что данное преобразование унитарно и представляет собой последовательность двух действий в фазовом пространстве: зависящей от времени дилатации конфигурационного пространства и трансляции импульса, линейно зависящей от канонической пространственной переменной. Композицию этих двух действий можно определить с помощью унитарных операторов $U_{2}=e^{i \Delta_{2}(t)(p q+q p)}$ 
и $U_{1}=e^{\Delta_{1}(t) q^{2}}$ соответственно. Важно то, что композиция двух зависящих от времени действий такого типа может привести к ослаблению влияния времени в неавтономных гамильтоновых квадратичных системах в том смысле, что в новых канонических координатах решение уравнения Шредингера в основном связано с решением не зависящих от времени спектральных задач (см., например, обсуждения в работах [8], [10], [13]). А именно, это происходит, если гамильтониан имеет вид

$$
H=\frac{p^{2}}{2 m(t)}+\frac{m(t) \omega(t)^{2}}{2} q^{2}
$$

при условии, что [13]

$$
U_{1}=\exp \left\{-i \frac{\dot{\rho}(t) \rho(t) m(t)}{2} q^{2}\right\}, \quad U_{2}=\exp \left\{\frac{i}{2} \ln [\rho(t)](p q+q p)\right\},
$$

где $\rho(t)$ зависит от потенциальной функции $\omega$ частотного типа и функции $m$ массового типа и эта зависимость определяется дифференциальным уравнением

$$
m(t) \ddot{\rho}(t)+\dot{m}(t) \dot{\rho}(t)+m(t) \omega(t)^{2} \rho(t)=\frac{1}{m(t) \rho(t)^{3}}
$$

(которое чаще преподносится как уравнение Ермакова для функции $\sigma=\kappa \sqrt{m} \rho$, где $\kappa=$ const, см., например, статью [14] и ссылки в ней). Таким образом, применяя преобразование (3), в котором коэффициенты

$$
A(t)=\rho(r)^{-1}, \quad B(t)=\rho(r), \quad C(t)=-m(t) \dot{\rho}(t)
$$

зависят от времени, получаем гамильтониан $H^{\prime}=\left(2 m \rho^{2}\right)^{-1}\left(P^{2}+Q^{2}\right)$. Теперь возникает вопрос, насколько далеко можно зайти, применяя данное преобразование к другим системам для получения нового применимого на практике гамильтониана. В простейшем случае он может принять форму произведения зависящего от времени гамильтониана на зависящий от времени множитель. На самом деле не следует априори отказываться от рассмотрения этого удобного случая. А именно, можно заметить, что если не являющийся квадратичным потенциал $V$, входящий в гамильтониан $H(1)$, удовлетворяет условию

$$
m(t) \rho^{2}(t) V(\rho(t) q, t)=W(q),
$$

то применение унитарного преобразования $U=U_{1} U_{2}$, где $U_{1,2}$ определены формулами (5), приводит к новому гамильтониану

$$
H^{\prime}=\frac{1}{m(t) \rho^{2}(t)} H_{0}^{\prime}, \quad \text { где } \quad H_{0}^{\prime}=\frac{P^{2}+Q^{2}}{2}+W(Q),
$$

в котором скейлинговая функция $\rho$ и массовая функция $m(t)$ связаны дифференциальным уравнением

$$
m(t) \ddot{\rho}(t)+\dot{m}(t) \dot{\rho}(t)=\frac{1}{m(t) \rho(t)^{3}} .
$$

В действительности для не являющихся квадратичными, как в выражении (4), потенциалов выясняется, что если потребовать, чтобы получающийся в результате 
гамильтониан был диффеоморфен по переменной времени автономному оператору, то условие (6) разделяется на два разных условия: алгебраическое ограничение (8), позволяющее выделить класс потенциалов, для которых можно решить рассматриваемую задачу, и дифференциальное уравнение (10), позволяющее найти подходящую скейлинговую функцию $\rho$, порождающую искомое каноническое преобразование. В случае квадратичных потенциалов (4) оба условия сливаются в одно, поскольку оказывается, что они оба связаны с коэффициентами квадратичных пространственных членов $Q^{2}$, порождаемых преобразованием. Рассуждая с несколько иной точки зрения, можно утверждать, что система, описываемая гамильтонианом (1), имеет динамический инвариант $I=U H_{0}^{\prime} U^{-1}$. В самом деле, подходящие условия для коэффициентов в формуле (3) определяются таким образом, что замена переменных $(q, p, t) \leftrightarrow(Q, P, \tau)$ позволяет записать динамическую переменную $I$ как стационарную, зависящую от фазовой координаты $(Q, P)$ (т. е. $I(p, q, t) \rightarrow I(P, Q))$. В частности, этого можно достичь, если задать коэффициенты согласно выражениям (7) и осуществить замену переменной времени вида

$$
\tau=\frac{1}{2} \int_{t_{0}}^{t} \frac{1}{m\left(t^{\prime}\right) \rho\left(t^{\prime}\right)^{2}} d t^{\prime}
$$

\section{3. УПОРЯДОЧЕННОЕ ПО ВЕЙЛЮ РАЗЛОЖЕНИЕ В РЯД ОПЕРАТОРА $q^{n} / p^{m}$ ПРИ ЛИНЕЙНЫХ ПРЕОБРАЗОВАНИЯХ ФАЗОВОГО ПРОСТРАНСТВА}

В данном разделе мы подробно исследуем, каким образом линейные зависящие от времени канонические преобразования воздействуют на упорядоченное по Вейлю разложение в ряд оператора $q^{n} / p^{m}$, являющегося элементом так называемого базиса Бендера-Данна. Прежде чем начать рассуждения, вспомним определение упорядоченного по Вейлю операторного базиса Бендера-Данна.

3.1. Операторный базис Бендера-Данна в фазовом пространстве. Исследование операторных уравнений, связанных с коммутаторами, можно провести, применяя технику упорядочения некоммутирующих операторов (см., например, статьи [1]-[6] и ссылки в них). Поиск решения операторных уравнений в таком случае превращается в решение комбинаторной задачи, в процессе решения которой в действительности можно обнаружить интересные факты (см., например, результаты работ [5], [15]). В частности, метод решения основывается на введении упорядоченности по Вейлю, рассмотренной Бендером и Данном в их познавательных статьях [1]. Данный метод фактически представляет собой поиск решения уравнения относительно оператора $\widehat{F}(\hat{q}, \hat{p})$ посредством определения его составляющих в базисе $\left\{\widehat{T}_{m, n}\right\}_{m, n \in \mathbb{Z}}$. Элементы $\widehat{T}_{m, n}$ данного базиса можно построить путем упорядочения по Вейлю произведений $\hat{p}^{m} \hat{q}^{n}$ целых степеней канонических пространственных или импульсных операторов. Поскольку оператор ищется в виде разложения в ряд, $\widehat{F}=\sum_{m, n} f_{m, n} \widehat{T}_{m, n}$, элементы базиса $\left\{\widehat{T}_{m, n}\right\}$ определяются с помощью применения известной процедуры упорядочения операторов

$$
\widehat{T}_{m, n}=: \hat{p}^{m} \hat{q}^{n}:_{\mathrm{Wey} 1}=\frac{1}{2^{n}} \sum_{k=0}^{n}\left(\begin{array}{l}
n \\
k
\end{array}\right) \hat{q}^{k} \hat{p}^{m} \hat{q}^{n-k}=\frac{1}{2^{m}} \sum_{k=0}^{m}\left(\begin{array}{c}
m \\
k
\end{array}\right) \hat{p}^{j} \hat{q}^{n} \hat{p}^{n-j},
$$


где для отрицательных степеней импульсов $\hat{p}$ (координат $\hat{q}$ ) следует использовать первое (второе) по счету из приведенных выражений [1] (далее мы опускаем символ “А” в обозначениях операторов, для того чтобы упростить обозначения и поскольку это не приведет к возникновению неясностей). Отметим, что если показатели степеней $m$ или $n$ отрицательны, то следует записывать биномы через гамма-функции ${ }^{1)}$. Свойства базиса Бендера-Данна $\left\{\widehat{T}_{m, n}\right\}$ рассмотрены в статьях [15] и [17].

\section{2. Действие линейных зависящих от времени канонических преобра-} зований на базис Бендера-Данна. Каноническое преобразование $\mathcal{C}$ в фазовом пространстве естественным образом порождает преобразование динамических переменных в том же пространстве. Если преобразование зависит от времени, непосредственный квантовый аналог классической динамической переменной можно получить посредством зависящих от времени отображений, действующих на отдельные операторы, входящие в ее определение. В частности, если каноническое преобразование $\mathcal{C}$ применить к элементам базиса Бендера-Данна $T_{m, n}$, то упорядоченный по

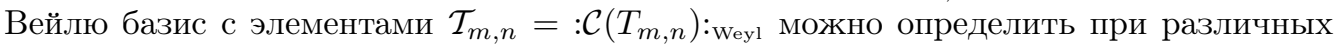
временах. В данном пункте, используя линейное преобразование пространственной переменной и импульса, мы определяем явный вид представления операторов $\mathcal{T}_{m, n}$ в базисе $\left\{T_{m, n}\right\}$. Рассмотрим сначала наиболее общий случай, когда преобразования имеют вид

$$
Q=A q+D p, \quad P=B p+C q
$$

(при $A B-C D=1$ они являются каноническими), и рассмотрим теперь каноническое преобразование, вытекающее из выражений (3), в котором зависящие от времени коэффициенты задаются формулами (7):

$$
Q=\frac{q}{\rho(t)}, \quad P=\rho(t) p-m(t) \dot{\rho}(t) q .
$$

Для достижения нашей цели воспользуемся следующим подходом, основанным на пошаговых вычислениях. Введем базис Бендера-Данна, связанный с парой новых канонических операторов $Q$ и $P$, т. е. операторный базис, состоящий из упорядоченных по Вейлю элементов

$$
\mathcal{T}_{-m, n}=: P^{-m} Q^{n}:_{\text {Weyl }}=\frac{1}{2^{n}} \sum_{k=0}^{n}\left(\begin{array}{l}
n \\
k
\end{array}\right) Q^{k} P^{-m} Q^{n-k} .
$$

Мы хотели бы найти выражение в замкнутой форме для операторов (15) в терминах начальных переменных $q, p$ и $t$. Если задать каноническое преобразование (13), то в самом деле можно заметить, что элементы $\mathcal{T}_{-m, n}$ зависят от исходных переменных $(q, p, t)$. В частности, можно определить $\mathcal{T}_{-m, n}$ с помощью упорядоченных по Вейлю разложений различных степеней переменных $q$ и $p$. Если в $(13) C=0$, то такое представление очевидно, поскольку в этом случае применение канонического преобразования подразумевает всего лишь применение зависящего от времени масштабирования пространственной переменной и импульса; соответственно, $\mathcal{T}_{-m, n} \rightarrow b^{m+n} T_{-m, n}$.

\footnotetext{
1) Заметим, что здесь могут возникнуть вполне очевидные вопросы, касающиеся формальных определений и того, как действуют пространственные и импульсные операторы, а также операторы, обратные к ним (см. исследования возможных следствий введения различных ограничений, например, в работах [2], [16]).
} 
Однако если $C \neq 0$, связь между двумя базисами $\left\{\mathcal{T}_{-m, n}\right\}$ и $\left\{T_{-j, k}\right\}$ уже не так тривиальна. В самом деле, хотя положительные степени оператора $Q$ легко можно выразить через переменные $(q, p, t)$ в виде

$$
Q^{\alpha}=(D p+A q)^{\alpha}=\sum_{s=0}^{\alpha}\left(\begin{array}{c}
\alpha \\
s
\end{array}\right) \frac{D^{\alpha-s} A^{s}}{2^{\alpha-s}} \sum_{u=0}^{\alpha-s}\left(\begin{array}{c}
\alpha-s \\
u
\end{array}\right) p^{u} q^{s} p^{\alpha-s-u}
$$

(и подставить в (15) при $\alpha=k$ и $\alpha=n-k$ ), для отрицательных степеней оператора $P$, которые выглядят как

$$
P^{-m}=C^{-m} p^{-m} \frac{1}{1+\left(\frac{C}{B}\right)^{m} \sum_{j=0}^{m-1} D_{m, j} T_{j, m-j} p^{-m}},
$$

ситуация усложняется. Представим операторы (15) в виде

$$
\begin{aligned}
\mathcal{T}_{-m, n}^{\mathcal{C}}= & \frac{D^{n}}{4^{n} C^{m}} \sum_{k=0}^{n}\left(\begin{array}{l}
n \\
k
\end{array}\right) \sum_{s=0}^{k}\left(\begin{array}{l}
k \\
s
\end{array}\right) \frac{2^{s} A^{s}}{D^{s}} \sum_{u=0}^{k-s}\left(\begin{array}{c}
k-s \\
u
\end{array}\right) \sum_{t=0}^{n-k} \frac{2^{t} A^{t}}{D^{t}} \sum_{v=0}^{n-k-t}\left(\begin{array}{c}
n-k-t \\
v
\end{array}\right) \times \\
& \times p^{u} q^{s} p^{k-s-u-m}\left(1+\frac{C^{m}}{B^{m}} \sum_{j=0}^{m-1} D_{m, j} T_{j, m-j} p^{-m}\right)^{-1} p^{v} q^{t} p^{n-k-t-v}
\end{aligned}
$$

(здесь и далее мы используем верхний индекс $\mathcal{C}$ для того, чтобы показать, что для записи операторов в терминах переменных $(q, p)$ было применено каноническое соотношение $(17))$. Обращаясь к разложению $\left(1+\Delta_{m}\right)^{-1}=\sum_{r=0}^{\infty}\left(-\Delta_{m}\right)^{r}$ для оператора

$$
\Delta_{m}=\frac{C^{m}}{B^{m}} \sum_{j=0}^{m-1} D_{m, j} T_{j, m-j} p^{-m}
$$

можно переписать формулу (18) как

$$
\begin{aligned}
\mathcal{T}_{-m, n}^{\mathcal{C}}= & \frac{D^{n}}{4^{n} C^{m}} \sum_{r=0}^{\infty}(-1)^{r} \sum_{k=0}^{n}\left(\begin{array}{l}
n \\
k
\end{array}\right) \sum_{s=0}^{k}\left(\begin{array}{l}
k \\
s
\end{array}\right) \frac{2^{s} A^{s}}{D^{s}} \sum_{u=0}^{k-s}\left(\begin{array}{c}
k-s \\
u
\end{array}\right) \times \\
& \times \sum_{t=0}^{n-k} \frac{2^{t} A^{t}}{D^{t}} \sum_{v=0}^{n-k-t}\left(\begin{array}{c}
n-k-t \\
v
\end{array}\right) \mathcal{O}_{k, m, n, s, t, u, v}^{r},
\end{aligned}
$$

где

$$
\mathcal{O}_{k, m, n, s, t, u, v}^{r}=p^{u} q^{s} p^{k-s-u-m} \Delta_{m}^{r} p^{v} q^{t} p^{n-k-t-v} .
$$

На данном этапе операторы $\mathcal{T}_{-m, n}^{\mathcal{C}}$ определяются в терминах последовательных действий произведений различных степеней исходных некоммутирующих канонических операторов $q$ и $p$. Для того чтобы как следует разобраться, как именно действуют операторы $q$ и $p$, можно формально упростить выписанные выше операторные ряды. Самый простой способ заключается в том, что мы вводим нормальное упорядочение на множестве операторов (21), применяя формулу

$$
q^{k} p^{\ell}=\sum_{\alpha=0}^{k} B_{\alpha, k, \ell} p^{\ell-\alpha} q^{k-\alpha}
$$


в которой

$$
\begin{array}{rlrl}
B_{\alpha, k, \ell} & =\frac{(-i \hbar)^{\alpha} \Gamma(k+1) \Gamma(\ell+1)}{\alpha ! \Gamma(k-\alpha+1) \Gamma(\ell-\alpha+1)}, & k \geqslant 0, & \ell>0, \\
B_{\alpha, k, \ell}=\frac{(-i \hbar)^{\alpha} \Gamma(k+1) \Gamma(\alpha-\ell)}{\alpha ! \Gamma(k-\alpha+1) \Gamma(-\ell)}, & k \geqslant 0, & \ell<0 .
\end{array}
$$

Согласно вышесказанному выражение (21) можно записать в виде

$$
\mathcal{O}_{k, m, n, s, t, u, v}^{r}=\frac{C^{m r}}{B^{m r}} \underbrace{\sum_{j_{i}=0}^{m-1} D_{m, j_{i}} \sum_{\alpha_{i}=0}^{\delta_{i}} B_{\alpha_{i}, \delta_{i}, \gamma_{i}}}_{i=1,2, \ldots, r+1} p^{n-(r+1) m+J_{i}-A_{i}-s-t} q^{r m-J_{i}-A_{i}+s+t},
$$

где

$$
\begin{gathered}
J_{i}=\sum_{\ell=1}^{i} j_{\ell}, \quad A_{i}=\sum_{\ell=0}^{i+1} \alpha_{\ell}, \\
\delta_{i}=\left\{\begin{array}{ll}
t, & i=0, \\
m-j_{i}, & 1 \leqslant i \leqslant r, \\
s, & i=r+1,
\end{array} \gamma_{i}= \begin{cases}n-k-t-v, & i=0, \\
n-i m-k-t+J_{i}-A_{i-2}-\beta_{i}, & 1 \leqslant i \leqslant r, \\
n-i m-s-t-u+J_{i-1}-A_{i-1}, & i=r+1,\end{cases} \right.
\end{gathered}
$$

и использовано обозначение

$$
\underbrace{\sum_{j_{i}=0}^{\alpha} F_{j_{i}}}_{i=1,2, \ldots, r}(\cdot)=\sum_{j_{1}=0}^{\alpha} F_{j_{1}} \times \sum_{j_{2}=0}^{\alpha} \times F_{j_{2}} \times \cdots \times \sum_{j_{r}=0}^{\alpha} F_{j_{r}}(\cdot) .
$$

Чтобы вернуться к базису $T_{m, n}$, определенному формулой (12), применим соотношение

$$
p^{a} q^{b}=T_{a, 0} T_{0, b}=\sum_{c=0}^{\infty} \sum_{d=0}^{j} F_{a, b, c, d} T_{a-c, b-c}
$$

где

$$
F_{a, b, c, d}=\left[\frac{1}{c !}\left(\frac{i}{2}\right)^{c}\right]\left[\frac{c !}{(c-d) ! d !}\right]\left[\frac{\Gamma(a+1) \Gamma(b+1)}{\Gamma(a+1-d) \Gamma(b+1-d) \Gamma^{2}(d-2 c)}\right],
$$

которое можно упростить в силу равенства

$$
p^{a} q^{b}=T_{a, 0} T_{0, b}=\sum_{c=0}^{b}\left(\frac{-i \hbar}{2}\right)^{c} \frac{\Gamma(1-a) \Gamma(1+b)}{\Gamma(1-a-j) \Gamma(1+b-j)} T_{a-c, b-c}
$$

в случае неположительных степеней оператора $p$ и неотрицательных степеней оператора q. Таким образом, мы получаем окончательный вид выражения (21):

$$
\mathcal{O}_{k, m, n, s, t, u, v}^{r}=\sum_{c=0}^{\infty} \sum_{d=0}^{j} \underbrace{\sum_{\alpha_{i}=0}^{\delta_{i}} B_{\alpha_{i}, \delta_{i}, \gamma_{i}}}_{i=1,2, \ldots, r+1} F_{a, b, c, d} T_{a-c, b-c},
$$

где $a=n-(r+1) m+J_{r}-A_{r}-s-t$ и $b=r m-J_{r}-A_{r}+s+t$, и завершаем исследование того, каким образом видоизменяются элементы упорядоченного по Вейлю базиса Бендера-Данна при линейных преобразованиях (13). 
3.2.1. Подкласс канонических преобразований (14). Рассмотрим теперь канонические преобразования (3), в которых зависящие от времени коэффициенты задаются формулами (7), т. е. канонические преобразования (14). В этом случае общий вид операторов $\mathcal{T}_{m, n}^{\mathcal{C}}$ упрощается:

$$
\mathcal{T}_{-m, n}^{\mathcal{C}}=\frac{1}{2^{n} \rho^{(m+n)}} \sum_{r=0}^{\infty}(-1)^{r} \sum_{k=0}^{n}\left(\begin{array}{l}
n \\
k
\end{array}\right) \mathcal{O}_{k, m, n}^{r}
$$

где

$$
\mathcal{O}_{k, m, n}^{r}=\left(\frac{-m \dot{\rho}}{\rho}\right)^{m r} q^{k} p^{-m}\left[\sum_{j=0}^{m-1} D_{m, j} T_{j, m-j} p^{-m}\right]^{r} q^{n-k}
$$

Согласно формуле (33) после некоторых преобразований мы можем записать для каждого оператора $\mathcal{O}_{k, m, n}^{r}$ выражение в замкнутой форме:

$$
\mathcal{O}_{k, m, n}^{r}=\left(\frac{-m \dot{\rho}}{\rho}\right)^{m r} \underbrace{\sum_{j_{i}=0}^{m-1} \frac{D_{m, j_{i}}}{2^{j_{i}}} \sum_{\beta_{i}=0}^{j_{i}}\left(\begin{array}{c}
j_{i} \\
\beta_{i}
\end{array}\right) \sum_{\alpha_{i}=0}^{\delta_{i}} B_{\alpha_{i}, \delta_{i}, \gamma_{i}}}_{i=1,2, \ldots, r+1} p^{-(r+1) m+d_{r}} q^{n+r m-s_{r}},
$$

где

$$
\begin{gathered}
d_{r}=\sum_{\ell=1}^{r+1}\left(j_{\ell}-\alpha_{\ell}\right), \quad s_{r}=\sum_{\ell=1}^{r+1}\left(j_{\ell}+\alpha_{\ell}\right), \\
\delta_{i}=m-j_{i}, \quad i=1,2, \ldots, r, \quad \delta_{r+1}=k
\end{gathered}
$$

и

$$
\gamma_{i}=\sum_{\ell=1}^{i} j_{\ell}-\sum_{\ell=1}^{i-1} \alpha_{\ell}-\beta_{i}-i m=d_{i-2}+j_{i}-\beta_{i}-i m
$$

(мы предположили, что $\sum_{\alpha=n_{0}}^{n}=0$ при $n<n_{0}$ и $\beta_{r+1}=j_{r+1}=0$ ). Поэтому, принимая во внимание равенство (30), получаем из соотношения (34) формулу

$$
\begin{aligned}
\mathcal{O}_{k, m, n}^{r}= & \left(\frac{-m \dot{\rho}}{\rho}\right)^{m r} \underbrace{\sum_{j_{i}=0}^{m-1} \frac{D_{m, j_{i}}}{2^{j_{i}}} \sum_{\beta_{i}=0}^{j_{i}}\left(\begin{array}{c}
j_{i} \\
\beta_{i}
\end{array}\right) \sum_{\alpha_{i}=0}^{\delta_{i}} B_{\alpha_{i}, \delta_{i}, \gamma_{i}}}_{i=1,2, \ldots, r+1} \times \\
& \times \sum_{c=0}^{b}\left(\frac{-i \hbar}{2}\right)^{c} \frac{\Gamma(1-a) \Gamma(1+b)}{\Gamma(1-a-j) \Gamma(1+b-j)} T_{a-c, b-c},
\end{aligned}
$$

в которой $a=-(r+1) m+d_{r}, b=n+r m-s_{r}$. Поскольку $a$ всегда отрицательно, можно воспользоваться тождеством

$$
\frac{\Gamma(1-a)}{\Gamma(1-a-j)}=(-1)^{j} \frac{(-a-1+j) !}{(-a-1) !}, \quad a<0,
$$


и осуществить в $(38)$ замену переменной $a \rightarrow-a$. Она приводит к формуле

$$
\begin{aligned}
\mathcal{O}_{k, m, n}^{r}= & \frac{(-m \dot{\rho})^{m r}}{\rho^{m(r+1)}} \underbrace{\sum_{j_{i}=0}^{m-1} \frac{D_{m, j_{i}}}{2^{j_{i}}} \sum_{\beta_{i}=0}^{j_{i}}\left(\begin{array}{c}
j_{i} \\
\beta_{i}
\end{array}\right) \sum_{\alpha_{i}=0}^{\delta_{i}} B_{\alpha_{i}, \delta_{i}, \gamma_{i}}}_{i=1,2, \ldots, r+1} \times \\
& \times \sum_{c=0}^{b}\left(\frac{i \hbar}{2}\right)^{c} \frac{(a+j-1) ! b !}{(b-j) !(a-1) !} T_{-a-c, b-c},
\end{aligned}
$$

где теперь $a=(r+1) m-d_{r}, b=-n-r m+s_{r}$. В свою очередь из этого следует, что операторы $\mathcal{T}_{m, n}^{\mathcal{C}}$ в (32) выражаются через операторы базиса Бендера-Данна (12) посредством соотношения

$$
\begin{aligned}
\mathcal{T}_{m, n}^{\mathcal{C}}= & \frac{1}{2^{n}} \sum_{r=0}^{\infty} \frac{(-1)^{r}(-m \dot{\rho})^{m r}}{\rho(t)^{n+m(r+1)}} \sum_{k=0}^{n}\left(\begin{array}{l}
n \\
k
\end{array}\right) \underbrace{\sum_{j_{i}=0}^{m-1} \frac{D_{m, j_{i}}}{2^{j_{i}}} \sum_{\beta_{i}=0}^{j_{i}}\left(\begin{array}{c}
j_{i} \\
\beta_{i}
\end{array}\right) \sum_{\alpha_{i}=0}^{\delta_{i}} B_{\alpha_{i}, \delta_{i}, \gamma_{i}}}_{i=1,2, \ldots, r+1} \times \\
& \times \sum_{c=0}^{b}\left(\frac{i \hbar}{2}\right)^{c} \frac{(a+j-1) ! b !}{(b-j) !(a-1) !} T_{-a-c, b-c .}
\end{aligned}
$$

Структура формулы (40) весьма сильно отличается от структуры формулы, в которой фигурируют полиномы, содержащие произведения положительных степеней координатных и импульсных операторов, подвергшихся воздействию линейных преобразований [11]. В частности, это отличие отражено в бесконечности операторных сумм.

3.2.2. Действие канонических преобразований (14) на оператор $\mathcal{T}_{-n, n}$. Принимая во внимание первые результаты, полученные с применением формальных разложений в упорядоченном по Вейлю базисе (12), например результаты, касающиеся фундаментального случая гармонического осциллятора, полученные Бендером и Данном в статьях [1], а также вспоминая, каким образом, используя линейные канонические преобразования [10], можно естественным образом расширить эти результаты на случай зависимости от времени, в настоящем пункте мы сосредотачиваем свое внимание на ситуации, когда $m=n$. Более того, поскольку минимальное решение для наблюдаемой, сопряженной с квадратичным гамильтонианом, совпадает с квантованной по Вейлю формой решения в классической задаче, для наших целей достаточно взять формулу (40) в пределе в классическом смысле. Для рассматриваемого случая $m=n$ член нулевого порядка по $\hbar$ соответствует случаю $\alpha_{j}=0$ $(j=1,2, \ldots, r)$ и $c=0$ в выражении (39). Данный критерий существенно упрощает формулу для оператора $\mathcal{T}_{-n, n}^{\mathcal{C}}$, приводя к выражению

$$
\begin{aligned}
\mathcal{T}_{-n, n}^{\mathcal{C}}= & \rho^{-2 n} T_{-n, n}+\rho^{-2 n} \sum_{r=1}^{\infty}(-1)^{r}\left(\frac{-m \dot{\rho}}{\rho}\right)^{n r} \times \\
& \times \underbrace{\sum_{j_{i}=0}^{n-1}\left(\begin{array}{c}
n \\
j_{i}
\end{array}\right)\left(\frac{-\rho}{m \dot{\rho}}\right)^{j_{i}}}_{r \text { раз, } 1 \leqslant i \leqslant r} T_{-(r+1) n+\sum_{i=1}^{r} j_{i},(r+1) n-\sum_{i=1}^{r} j_{i}},
\end{aligned}
$$


которое можно записать в более простой форме

$$
\mathcal{T}_{-n, n}^{\mathcal{C}}=\rho^{-2 n} T_{-n, n}+\frac{1}{(m \rho \dot{\rho})^{n}} \sum_{r=n+1}^{\infty}(-1)^{r}\left(\begin{array}{l}
r-1 \\
n-1
\end{array}\right)\left(\frac{-m \dot{\rho}}{\rho}\right)^{r} T_{-r, r}
$$

где член, соответствующий случаю $r=0$, был выделен для того, чтобы можно было напрямую сравнить это выражение с выражением до применения преобразования. В действительности полезно отметить, что ряды (41), (42) также можно преобразовать, откуда окончально записываем равенство

$$
\mathcal{T}_{-n, n}^{\mathcal{C}}=\frac{X^{n}}{2 \rho^{2 n}}\left[1-\frac{m \dot{\rho}}{\rho} X\right]^{-n}+\frac{Y^{n}}{2 \rho^{2 n}}\left[1-\frac{m \dot{\rho}}{\rho} Y\right]^{-n}
$$

где $X$ и $Y$ суть операторы, имеющие вид

$$
X=\frac{1}{p} q, \quad Y=q \frac{1}{p} .
$$

В самом деле, последние равенства можно получить, непосредственно применяя полезные тождества

$$
T_{-r, r}=\frac{1}{2}\left(\frac{1}{p} q\right)^{r}+\frac{1}{2}\left(q \frac{1}{p}\right)^{r}=\frac{1}{2} X^{r}+\frac{1}{2} Y^{r}
$$

и

$$
\sum_{r=n+1}^{\infty}(-1)^{r}\left(\begin{array}{l}
r-1 \\
n-1
\end{array}\right) z^{r}=z^{n}\left(-1+(1-z)^{-n}\right)
$$

Собственно говоря, использование равенства (43) может оказаться целесообразным при некоторых исследованиях.

\section{4. КЛАССИЧЕСКИЕ И КВАНТОВЫЕ РАЗЛОЖЕНИЯ НАБЛЮДАЕМЫХ ПО КООРДИНАТНЫМ ПЕРЕМЕННЫМ И ИМПУЛЬСАМ}

В следующих разделах мы рассматриваем несколько примеров применения упорядоченного по Вейлю базиса типа Бендера-Данна при исследовании интегрируемости неавтономных систем. Но прежде мы хотели бы сделать несколько замечаний касательно надежности процедур квантования и деквантования в свете рассматриваемой задачи. Действительно, непосредственное квантование классических решений в фазовом пространстве в большинстве случаев оказывается нерезультативным (за некоторыми исключениями, рассмотренными далее), поскольку невозможно представить решение квантовой версии уравнения, полученное из алгебраических соображений, просто заменяя скобки Пуассона на коммутаторы. Квантовые операторные уравнения требуют непосредственного исследования, даже если имеется решение их классического аналога. При превращении классической динамической переменной в квантовую возникают трудности, обусловленные невозможностью аккуратного перенесения всех алгебраических свойств решений. Основная причина этих трудностей заключается в том, что при классическом рассмотрении в отличие 
от рассмотрения на квантовом уровне отсутствуют законы упорядочения: разностное уравнение для коэффициентов разложения, которое можно получить в классическом базисе классического динамического объекта, подчиняющемся алгебраическому условию (в смысле алгебры скобок Пуассона), обычно отличается от разностного уравнения, которое можно получить для коэффициентов разложения в квантовом аналоге классического базиса для наблюдаемой, который удовлетворяет условию, получающемуся после замены скобок Пуассона на коммутаторы. Если мы рассматриваем классическую наблюдаемую, представленную в одном базисе (например, базисе на основе набора произведений степеней координатных переменных и импульсов $t_{m, n}=p^{m} q^{n}$, связанного с разложениями типа лорановских), связи между коэффициентами структурно проще, поскольку с помощью формализма скобок Пуассона можно описать лишь те алгебраические аспекты, которые касаются скалярных функций и их производных, т. е. коммутативных объектов. Другими словами, в то время как прямое деквантование операторных уравнений и их решений приводит к выражениям, свойственным решению классической задачи, обратное утверждение неверно: непосредственное квантование решения классического уравнения для наблюдаемой не приводит к вычленению всех особенностей, свойственных решению квантовой версии динамического уравнения. Для произвольной динамической переменной данный момент чрезвычайно важен, и мы переходим к его обсуждению. Чтобы эффективно проиллюстрировать нашу точку зрения, достаточно рассмотреть случай наблюдаемых, канонически сопряженных с гамильтонианом в простом случае автономной системы, когда квантовая (классическая) динамика управляется степенным потенциалом, а именно квантовым (классическим) гамильтонианом

$$
H_{L}=\frac{p^{2}}{2}+\frac{q^{L}}{L}
$$

где $L$ - натуральное число. Однако данное рассуждение можно немедленно распространить на другие случаи наблюдаемых и на класс неавтономных аналитических потенциалов. Вследствие этого рассмотрим формальное разложение по базису Бендера-Данна

$$
\Theta_{L}=\sum_{m, n} \alpha_{m, n} T_{m, n}
$$

для оператора $\Theta_{L}$, канонически сопряженного с $H_{L}$ посредством операторного уравнения $\left[\Theta_{L}, H_{L}\right]=i \hbar$. В алгебре операторов $T_{m, n}$ верно равенство

$$
\begin{aligned}
& {\left[T_{m, n}, T_{r, s}\right]=2 \sum_{j=0}^{\infty} \frac{1}{(2 j+1) !}\left(\frac{i \hbar}{2}\right)^{2 j+1} \sum_{l=0}^{2 j+1}(-1)^{l}\left(\begin{array}{c}
2 j+1 \\
l
\end{array}\right) \times} \\
& \quad \times \frac{\Gamma(m+1) \Gamma(n+1) \Gamma(r+1) \Gamma(s+1)}{\Gamma(m-l+1) \Gamma(n+l-2 j) \Gamma(r+l-2 j) \Gamma(s-l+1)} T_{m+r-2 j-1, n+s-2 j-1}= \\
& =i \hbar(n r-m s) T_{m+r-1, n+s-1}+2 \sum_{j=1}^{\infty}\left(\frac{i \hbar}{2}\right)^{2 j+1} \frac{\Gamma(n+1) \Gamma(r+1)}{(2 j+1) ! \Gamma(n-2 j) \Gamma(r-2 j)} \times \\
& \quad \times_{3} F_{2}[-1-2 j,-m,-s ;-2 j+n,-2 j+r ; 1] T_{m+r-2 j-1, n+s-2 j-1},
\end{aligned}
$$


поэтому

$$
\begin{gathered}
{\left[T_{m, n}, p^{2}\right]=2 i \hbar n T_{m+1, n-1},} \\
{\left[T_{m, n}, q^{L}\right]=-i \hbar L m T_{m-1, n+L-1}-i \hbar \sum_{j=1}^{\lfloor(L-1) / 2\rfloor} \hbar^{2 j} A_{m, j, L} T_{m-2 j-1, n+L-2 j-1},}
\end{gathered}
$$

где не равные нулю коэффициенты $A_{m, j, L}$ можно получить из формулы $(49)$ при $r=0$ и $s=L$ :

$$
A_{m, j, L}=\frac{(-1)^{j} \Gamma(m+1) \Gamma(L+1)}{4^{j}(2 j+1) ! \Gamma(m-2 j) \Gamma(L-2 j)} .
$$

Таким образом, квантовое каноническое отношение сопряженности $\left[\Theta_{L}, H_{L}\right]=i \hbar$ выполняется, если коэффициенты, характеризующие реализацию наблюдаемой $\Theta_{L}$, удовлетворяют рекуррентному выражению

$$
\begin{aligned}
& (n+1) \alpha_{m-1, n+1}-(m+1) \alpha_{m+1, n-L+1}- \\
& -\frac{1}{L} \sum_{j=1}^{\lfloor(L-1) / 2\rfloor} \hbar^{2 j} \alpha_{m+2 j+1, n-L+2 j+1} A_{m+2 j+1, n-L+2 j+1, L, j}=\delta_{m, n} \delta_{n, 0}
\end{aligned}
$$

(здесь использовано равенство $\left.1=\sum_{m, n} \delta_{m, 0} \delta_{n, 0} T_{m, n}\right)$. Рассматривая теперь проблему с классической точки зрения и применяя классический аналог базиса Бендера-Данна в фазовом пространстве, мы получаем разложение переменной $\Theta_{L}^{c}$, сопряженной с классическим гамильтонианом $H_{L}$, по координатной паре $(p, q)$ :

$$
\Theta_{L}^{\mathrm{c}}=\sum_{m, n} \alpha_{m, n}^{\mathrm{c}} t_{m, n}=\sum_{m, n} \alpha_{m, n}^{\mathrm{c}} p^{m} q^{n}
$$

Отсюда следует, что классическое условие канонического спаривания, связанное со скобкой Пуассона $\left\{\Theta_{L}^{\mathrm{c}}, H_{L}^{\mathrm{c}}\right\}=p \partial_{q} \Theta_{L}^{\mathrm{c}}-q^{L-1} \partial_{p} \Theta_{L}^{\mathrm{c}}=1$, порождает разностное уравнение

$$
(n+1) \alpha_{m-1, n+1}^{\mathrm{c}}-(m+1) \alpha_{m+1, n-s+1}^{\mathrm{c}}=\delta_{m, 0} \delta_{n, 0}
$$

для коэффициентов $\alpha_{m, n}^{\mathrm{c}}$. Таким образом, непосредственно квантуя динамическую переменную $\Theta_{L}^{c}$ и просто заменяя базисные элементы $t_{m, n}$ на их квантовые аналоги $T_{m, n}$, мы не получим всего набора свойств квантового аналога этой динамической переменной из-за квантового упорядочения. На самом деле, хотя деквантование наблюдаемой, являющейся решением соответствующего квантового уравнения, естественным образом приводит к ее классическому аналогу, который можно получить, формально решая исходное уравнение, заменяя коммутаторы на скобки Пуассона, при проведении обратной процедуры непосредственного квантования классической наблюдаемой можно упустить некоторые свойства.

\section{5. ПРИМЕРЫ. І. ЗАВИСЯЩИЕ ОТ ВРЕМЕНИ КВАДРАТИЧНЫЕ ГАМИЛЬТОНОВЫ СИСТЕМЫ}

Как и было задумано, в настоящем и следующих разделах мы рассматриваем несколько примеров применения упорядоченного по Вейлю базиса типа БендераДанна при исследовании интегрируемости квантовых неавтономных систем в случаях, когда для потенциала выполняется условие (8). Речь идет о случаях, когда 
зависимость потенциала от пространственной переменной определяется степенным членом или рядом, в котором зависящие от времени коэффициенты характеризуются степенным множителем той же степени, что и зависящие от пространственной переменной коэффициенты. Наше исследование ограничивается первым случаем. А именно, общая постановка рассматриваемой задачи имеет следующий вид. Пусть задана неавтономная гамильтонова система (1) и пусть $I \equiv I(q, p, t)$ - инвариантный оператор для этой системы. Как выглядит оператор $\Theta \equiv \theta(q, p, t)$, формально сопряженный с $I$ посредством уравнения коммутации

$$
[I, \Theta]=i \hbar,
$$

и каким образом можно записать $\Theta$ в упорядоченном по Вейлю базисе пространственных и импульсных операторов?

Чтобы ответить на эти вопросы, воспользуемся рассуждениями и замечаниями из предыдущих разделов. В частности, решения уравнения (55) можно записать в зависящем от времени базисе.

Мы начинаем обсуждение примеров с рассмотрения зависящего от времени гамильтониана

$$
H=\frac{p^{2}}{2 m(t)}+\frac{1}{2} m(t) \omega(t)^{2} q^{2},
$$

который можно преобразовать в новый неавтономный гамильтониан

$$
H^{\prime}=\frac{1}{2 m \rho^{2}} H_{0}, \quad H_{0}=P^{2}+Q^{2},
$$

с помощью линейного канонического преобразования вида (3). Инвариантный опеpaтор $I(q, p, t)=U H_{0} U^{-1}$ можно с учетом контекста записать как

$$
I(q, p, t)=\rho^{2} p^{2}-m \rho \dot{\rho}(p q+q p)+\left(m^{2} \dot{\rho}^{2}+\rho^{-2}\right) q^{2} .
$$

Операторная функция $\Theta(q, p, t)$, канонически сопряженная с $I(q, p, t)$, представляется в базисе $T_{r, s}$ разложением

$$
\Theta(q, p, t)=\sum_{r, s} \alpha_{r, s}(t) T_{r, s} .
$$

В результате мы получаем рекуррентное соотношение для коэффициентов $\alpha_{r, s}$ в (59):

$$
\rho^{2}(s+1) \alpha_{r-1, s+1}-\left(m^{2} \dot{\rho}^{2}+\rho^{-2}\right)(r+1) \alpha_{r+1, s-1}-(s-r) m \rho \dot{\rho} \alpha_{r, s}=\frac{1}{2} \delta_{r, 0} \delta_{s, 0} .
$$

Мы хотим найти минимальное решение уравнения (60). Под минимальным решением понимается решение при начальном приближении $\alpha_{-1,1}=1 /\left(2 \rho^{2}\right)$ и $\alpha_{r, s}=0$ при $r \leqslant 2$ и произвольных $s$. Для определения минимального решения мы сначала сводим рекуррентное соотношение (60) к рекуррентному выражению, содержащему один индекс, путем замены $s=k=-r$. Переопределяя коэффициенты согласно выражению

$$
A_{k}=\alpha_{r-1, s+1}=\alpha_{-k-1, k+1},
$$

мы приводим уравнение (60) относительно коэффициентов $A_{k}$ к виду

$$
\rho^{2}(k+1) A_{k}+\left(m^{2} \dot{\rho}^{2}+\rho^{-2}\right)(k-1) A_{k-2}-2 k m \rho \dot{\rho} A_{k-1}=0
$$


с начальным условием $A_{0}=1 / 2 \rho^{2}$. Уравнение (62) превращается в рекуррентное соотношение с постоянными коэффициентами

$$
\rho^{2} B_{k}+\left(m^{2} \dot{\rho}^{2}+\rho^{-2}\right) B_{k-2}-2 m \rho \dot{\rho} B_{k-1}=0, \quad B_{0}=\frac{1}{2 \rho^{2}},
$$

после замены $A_{k}=B_{k} /(k+1)$. Для того чтобы решить уравнение $(63)$, предположим, что решение имеет вид $B_{k}=x^{k}$ при некотором значении $x$. Для определения значений $x$ подставим это выражение в уравнение (63) и найдем два его корня

$$
x_{ \pm}=\frac{m(t) \rho \dot{\rho} \pm i}{\rho^{2}} .
$$

Тогда решение уравнения (63) в общем виде записывается как $B_{k}=c_{+} x_{+}^{k}+c_{-} x_{-}^{k}$, где $c_{+}$и $c_{-}$можно определить, используя начальные условия $B_{0}=1 / 2 \rho^{2}$ и $B_{1}=$ $m(t) \dot{\rho} / \rho^{3}$. Таким образом, находим, что $c_{ \pm}=(1 \pm m(t) \rho \dot{\rho}) / 4 \rho^{2}$. Окончательное выражение для оператора $\Theta(q, p, t)$ имеет вид

$$
\Theta(p, q, t)=\frac{1}{4 \rho^{2}} \sum_{k=0}^{\infty} A_{k} T_{-k, k}
$$

где коэффициенты $A_{k}$ определяются равенством

$$
A_{k}=\frac{(1+m \rho \dot{\rho})^{k+1}+(-1)^{k}(1-m \rho \dot{\rho})^{k+1}}{(k+1) \rho^{2 k}} .
$$

Вместо того чтобы решать уравнение (60), мы могли воспользоваться результатами раздела 3, а именно:

1) решить в базисе $\mathcal{T}_{m, n}$ более простое рекуррентное уравнение для оператора $\Theta_{0}(Q, P)=\sum_{m, n} \tilde{\alpha}_{m, n} \mathcal{T}_{m, n}$, канонически сопряженного с гамильтонианом $H_{0}$ в $(57)$, имеющее вид

$$
(n+1) \tilde{\alpha}_{m-1, n+1}-(m+1) \tilde{\alpha}_{m+1, n-1}=\delta_{m, 0} \delta_{n, 0},
$$

и получить решение (см. статьи [1], [5])

$$
\Theta_{0}=\sum_{k=0}^{\infty} \frac{(-1)^{k}}{2 k+1} \mathcal{T}_{-2 k-1,2 k+1}
$$

2) выразить базисные элементы $\mathcal{T}_{-2 k-1,2 k+1}$ в (68) через базисные элементы $T_{m, n}$ в $(q, p)$-представлении, полученные из формулы $(42)$ при $n=2 k+1$. Тогда разложение оператора $\Theta$ записывается как

$$
\Theta=\sum_{k=0}^{\infty} \frac{(-1)^{k}}{2 k+1}\left[\rho^{-4 k-2} T_{-2 k-1,2 k+1}+\frac{1}{(m \rho \dot{\rho})^{2 k+1}} \sum_{r=2 k+2}^{\infty}(-1)^{r}\left(\begin{array}{c}
r-1 \\
2 k
\end{array}\right)\left(\frac{-m \dot{\rho}}{\rho}\right)^{r} T_{-r, r}\right]
$$

или согласно (43) как

$$
\Theta=\frac{1}{2} \operatorname{arctg}\left[\frac{X}{\rho^{2}}\left(1-\frac{m \dot{\rho}}{\rho} X\right)^{-1}\right]+\frac{1}{2} \operatorname{arctg}\left[\frac{Y}{\rho^{2}}\left(1-\frac{m \dot{\rho}}{\rho} Y\right)^{-1}\right] .
$$

Легко проверить, что коэффициенты при каждом элементе $T_{-n, n}$ в выражениях (65), (66) и (69) совпадают. 


\section{6. ПРИМЕРЫ. II. ЗАВИСЯЩИЕ ОТ ВРЕМЕНИ ЛИНЕЙНЫЕ ГАМИЛЬТОНОВЫ СИСТЕМЫ}

В настоящем разделе мы рассматриваем систему с неавтономным гамильтониаHOM

$$
H=\frac{1}{2}\left[z(t) p^{2}+\frac{z(t)}{2 \rho(t)^{3}} q\right],
$$

который посредством канонического линейного преобразования (3) с коэффициентами (7) можно превратить в гамильтониан

$$
H^{\prime}=\frac{z(t)}{2 \rho(t)^{2}} H_{0}, \quad \text { где } \quad H_{0}=P^{2}+Q^{2}+Q .
$$

Гамильтонова формулировка динамической задачи, описываемой уравнением (71), диффеоморфна по переменной времени автономной системе, гамильтониан которой имеет вид $H_{0}=P^{2}+Q^{2}+Q$. Иными словами, можно описать рассматриваемый случай с помощью модели гармонического осциллятора с точностью до постоянного сдвига координаты $Q$ (и энергии): $Q \rightarrow \widetilde{Q}=Q+1 / 2$ (и $H_{0}=P^{2}+Q^{2}+Q \rightarrow P^{2}+$ $\widetilde{Q}^{2}-1 / 4$ соответственно). Следовательно, для системы с гамильтонианом (71) можно найти имеющие физический смысл решения операторных динамических уравнений, приспособив решения уравнений в модели гармонического осциллятора. Выбрать минимальные решения можно также на основе рассуждений в модели гармонического осциллятора. Далее для полноты и единообразия изложения, а также по причине наглядности мы проводим прямой вывод решения условия интегрируемости для системы (71), используя операторную форму гамильтониана (72) и не обращаясь к решению задачи в случае чисто квадратичного гамильтониана. Для начала на основе изложенного выше выпишем инвариант. Применяя обратное преобразование к гамильтониану $H_{0}$ в (72), получаем для гамильтониана (71) динамический инвариант

$$
I=\rho^{2}(t) p^{2}+\beta(t) q^{2}-\gamma(t)(p q+q p)+\rho^{-1}(t) q,
$$

где

$$
\beta(t)=\rho(t)^{-2}+\frac{\dot{\rho}(t)^{2}}{z(t)^{2}}, \quad \gamma(t)=\frac{\dot{\rho}(t) \rho(t)}{z(t)} .
$$

Вследствие этого, записывая в терминах разложений Вейля в базисе Бендера-Данна $\left\{T_{m, n}\right\}$ разложение решения для оператора $\Theta$, формально сопряженного с оператором (73) посредством уравнения (55), мы приходим к разностному уравнению

$\rho(t)^{2}(n+1) \alpha_{m-1, n+1}-\beta(t) \alpha_{m+1, n-1}-\gamma(t)(n-m) \alpha_{m, n}-\frac{1}{2 \rho(t)}(m+1) \alpha_{m+1, n}=\frac{1}{2} \delta_{m, 0} \delta_{n, 0}$

для зависящих от времени коэффициентов $\alpha_{m, n}$ в разложении $\Theta=\sum_{m, n} \alpha_{m, n} T_{m, n}$. $\mathrm{K}$ этому решению можно прийти, рассматривая гамильтониан $H_{0}=P^{2}+Q^{2}+Q$ и решая квантовое условие интегрируемости $\left[\Theta_{0}, H_{0}\right]=i \hbar$ в терминах разложений Вейля в базисе $\left\{\mathcal{T}_{m, n}\right\}$. А именно, если мы положим $\Theta_{0}=\sum_{m, n} \tilde{\alpha}_{m, n} \mathcal{T}_{m, n}$, то уравнение $\left[\Theta_{0}, H_{0}\right]=i \hbar$ приводит к разностному уравнению

$$
2(n+1) \tilde{\alpha}_{m-1, n+1}-2(m+1) \tilde{\alpha}_{m+1, n-1}-(m+1) \tilde{\alpha}_{m+1, n}=\delta_{m, 0} \delta_{n, 0}
$$


для зависящих от времени коэффициентов разложения $\tilde{\alpha}_{m, n}$. Минимальное решение соответствует выбору значений $\tilde{\alpha}_{-1,1}=1 / 2$ и $\tilde{\alpha}_{1,-1}=\tilde{\alpha}_{1,0}=0$. Используя метод обратной задачи Лиувилля, изложенный в статье [2] и вкратце рассмотренный в приложении, мы предполагаем, что общий вид оператора $\Theta$ описывается выражением

$$
\Theta_{0}=\sum_{k=0}^{\infty} \sum_{j=0}^{k} A_{k, j} \mathcal{T}_{-2 k-1,2 k-j+1}
$$

в котором требуется определить коэффициенты $A_{k, j}$. Из выражения (77) вытекает преобразование

$$
A_{k, j}=\tilde{\alpha}_{-2 k-1,2 k-j+1}=\tilde{\alpha}_{m-1, n+1},
$$

а также связь $A_{k, j}=0$ при $k<0$ и $j>k$. Это означает, что $k=-m / 2$ и $j=-m-n$ $(0 \leqslant j \leqslant k)$. Тогда новое рекуррентное соотношение имеет вид

$$
(2 k-j+1) A_{k, j}+(2 k-1) A_{k-1, j}+(2 k-1) A_{k-1, j-1}=\delta_{k, 0} \delta_{j, 0},
$$

и его решение задается формулой

$$
A_{k, j}=\frac{(-1)^{k}}{2 k+1}\left(\begin{array}{c}
2 k+1 \\
j
\end{array}\right)
$$

откуда следует, что

$$
\Theta_{0}=\sum_{k=0}^{\infty} \sum_{j=0}^{k} \frac{(-1)^{k}}{2 k+1}\left(\begin{array}{c}
2 k+1 \\
j
\end{array}\right) \mathcal{T}_{-2 k-1,2 k-j+1}
$$

Для решения зависящей от времени задачи нужно преобразовать базисные элементы в формуле (81) согласно равенству (40). Отметим, что в этом случае мы также можем применить соотношение

$$
\mathcal{T}_{-2 k-1,2 k+1-j}=\frac{i^{j}(2 k+1-j) !}{(2 k+1) !} \underbrace{\left[P,\left[P, \ldots\left[P, \mathcal{T}_{-2 k-1,2 k+1}\right] \ldots\right]\right]}_{j \text { раз }}
$$

и учесть тот факт, что формально

$$
\mathcal{T}_{-2 k-1,2 k+1-j}^{\mathcal{C}}=\frac{i^{j}(2 k+1-j) !}{(2 k+1) !} \underbrace{\left[\rho p-m \dot{\rho} q, \ldots\left[\rho p-m \dot{\rho} q, \mathcal{T}_{-2 k-1,2 k+1}^{\mathcal{C}}\right] \ldots\right]}_{j \text { раз }},
$$

где

$$
\begin{aligned}
& \mathcal{T}_{-2 k-1,2 k+1}^{\mathcal{C}}= \\
& =\left(\frac{-m \dot{\rho}}{\rho}\right)^{2 n+1} T_{-2 k-1,2 k+1}+\sum_{r=2 k+2}^{\infty}(-1)^{r} \frac{1}{\rho^{2 k+1}}\left(\frac{-m \dot{\rho}}{\rho}\right)^{r} \frac{\Gamma(r+2 k+1)}{\Gamma(r+1) \Gamma(2 k+1)} T_{-r, r} .
\end{aligned}
$$




\section{7. ПРИМЕРЫ. III. ЗАВИСЯЩИЕ ОТ ВРЕМЕНИ КВАДРАТИЧНЫЕ ГАМИЛЬТОНОВЫ СИСТЕМЫ}

В настоящем разделе мы детально исследуем квантовую интегрируемость неавтономных гамильтоновых систем с гамильтонианом

$$
H=\frac{z(t)}{2}\left[p^{2}+\frac{q^{4}}{2 \rho(t)^{6}}\right],
$$

используя рассуждения, приведенные в предыдущих разделах. Применяя каноническое координатное преобразование, рассматриваемое в настоящей статье, мы можем преобразовать гамильтониан (85) к виду

$$
H^{\prime}=\frac{z(t)}{2 \rho(t)^{2}} H_{0}, \quad H_{0}=\frac{P^{2}+Q^{2}}{2}+\frac{Q^{4}}{4} .
$$

Кроме того, динамический инвариант для гамильтониана (85) определяется выражением

$$
I=\eta(t) p^{2}+\beta(t) q^{2}-\gamma(t)(p q+q p)+\delta(t) q^{4},
$$

в котором $\eta(t)=\rho(t)^{2}, \delta(t)=\rho(t)^{-4}$ и $\beta(t), \gamma(t)$ выглядят так же, как и в случае линейного гамильтониана (т. е. $\beta(t)=\rho(t)^{-2}+\dot{\rho}(t)^{2} / z(t)^{2}$ и $\gamma(t)=\dot{\rho}(t) \rho(t) / z(t)$, см. формулу (74)). Нас интересуют решения условия интегрируемости $[\Theta, I]=i \hbar$, где $I$ определено согласно выражению (87). Поскольку решение ищется в виде $\Theta=\sum_{m, n} \alpha_{m, n} T_{m, n}$ в базисе $T_{m, n}$, мы записываем следующее рекуррентное соотношение для коэффициентов $\alpha_{m, n}$ :

$$
\begin{aligned}
2 \eta(t)(n & +1) \alpha_{m-1, n+1}-2 \beta(t)(m+1) \alpha_{m+1, n-1}-2 \gamma(t)(n-m) \alpha_{m, n}- \\
& -4 \delta(t)(m+1) \alpha_{m+1, n-3}+\delta(t)(m+3)(m+2)(m+1) \alpha_{m+3, n-1}= \\
& =\delta(t)(m+3)(m+2)(m+1) \alpha_{m+3, n-1} .
\end{aligned}
$$

Для того чтобы выбрать минимальное решение, при $m=n=0$ положим $\alpha_{1,-1}=$ $\alpha_{1,-3}=\alpha_{3,-1}=0$ и $\alpha_{-1,1}=1 / 2 \eta(t)$. Применяя преобразование

$$
\begin{gathered}
M=\frac{2 n+m+4 K-A}{6}, \quad N=\frac{n-m+2 K-2 A}{6}, \\
0 \leqslant A \leqslant(n+2) / 3, \quad 0 \leqslant K \leqslant(5 n+3 m-2) / 6,
\end{gathered}
$$

определим новую зависимую переменную

$$
C_{N, M, K, A}=\alpha_{-4 N+2 M-A-1,2 N+2 M-2 K+A+1}=\alpha_{m-1, n+1}
$$

и наложим ограничение $C_{N, M, K, A}=0$ при $N, M, K, A \leqslant 0, N \leqslant M \leqslant K$ и $A \leqslant$ $M+N-K+1$. Разностное уравнение для зависящих от времени коэффициентов $C_{N, M, K, A}(t)$ имеет вид

$$
\begin{aligned}
\eta(t) & (2 M+2 N-2 K+A+1) C_{N, M, K, A}+\beta(t)(4 N-2 M+A-1) C_{M-1, N-1, K-1, A}- \\
& -2 \gamma(t)(3 N+A-K) C_{N-1, M-1, K-1, A+1}+ \\
& +2 \delta(t)(4 N-2 M+A-1) C_{N-1, M-1, K, A}- \\
& -\delta(t)(4 N-2 M+A-1)(4 N-2 M+A-2)(4 N-2 M+A-3) C_{N-1, M, K, A}= \\
= & \frac{1}{2} \delta_{N, 0} \delta_{M, 0} \delta_{K, 0} \delta_{A, 0},
\end{aligned}
$$


поэтому

$$
\Theta=\sum_{N=0}^{\infty} \sum_{M=0}^{N} \sum_{K=0}^{M} \sum_{A=0}^{M+N-K+1} C_{N, M, K, A}(t) T_{-4 N+2 M-A-1,2 N+2 M-2 K+A+1} .
$$

С другой стороны, рассматривая в терминах координат $Q, P$ оператор $\Theta_{0}(Q, P)=$ $\sum_{m, n} \tilde{\alpha}_{m, n} \mathcal{T}_{m, n}$ и условие интегрируемости $\left[H_{0}, \Theta_{0}\right]=i \hbar$ для стационарного зависящего от квадрата потенциала гамильтониана $H_{0}$ в $(86)$, мы получаем рекуррентное ограничение

$$
\begin{gathered}
(n+1) \tilde{\alpha}_{m-1, n-1}-(m+1) \tilde{\alpha}_{m+1, n-1}+\frac{\hbar^{2}}{4}(m+1)(m+2)(m+3) \tilde{\alpha}_{m+3, n-1}- \\
-(m+1) \tilde{\alpha}_{m+1, n-3}=\delta_{m, 0} \delta_{n, 0} .
\end{gathered}
$$

Последний член в левой части выражения (89) отличает это выражение от формулы, которую можно получить для квадратичного осциллятора с гамильтонианом $H_{\mathrm{BD}}=$ $P^{2} / 2+Q^{4} / 4$, изученного Бендером и Данном в статье [1] (и позже исследованного Галапоном и Виллануэва в работе [2] с использованием метода обратной задачи Лиувилля). Стуктуру минимального оператора $\Theta_{0}$ можно задать формулой

$$
\Theta_{0}=\sum_{N=0}^{\infty} \sum_{M=0}^{N} \sum_{K=0}^{N-M} A_{M N K} \mathcal{T}_{-2 N-2 M-1,4 N-2 M-2 K+1}
$$

которая также является результатом применения метода обратной задачи Лиувилля (см. приложение). Осуществляя преобразование согласно равенствам $m=-2 M-2 N$ и $n=4 N-2 M-2 K$, можно связать коэффициенты $A_{M, N, K}$ с коэффициентами $\tilde{\alpha}_{m, n}$ посредством соотношения

$$
A_{M, N, K}=\alpha_{m-1, n+1}=\tilde{\alpha}_{-2 N-2 M-1,4 N-2 M-2 K+1}
$$

при условии, что $A_{N M K}=0, N, M, K \leqslant 0, N \leqslant M \leqslant K$. Таким образом, рекуррентное выражение (89) принимает вид

$$
\begin{aligned}
\delta_{M, 0} \delta_{N, 0} \delta_{K, 0}= & (4 N-2 M-2 K+1) A_{M, N, K}+(2 N+2 M-1) A_{M, N-1, K-1}- \\
& -\frac{\hbar^{2}}{4}(2 N+2 M-3)(2 N+2 M-2)(2 N+2 M-1) A_{M-1, N-1, K}+ \\
& +(2 N+2 M-1) A_{M, N-1, k} .
\end{aligned}
$$

Дальнейшее преобразование сводит разностное уравнение (теперь имеющее первый порядок по переменным $N, M, K)$ к уравнению, коэффициенты которого являются линейными функциями по $N, M, K$. Для этого определим

$$
B_{N M K}=\frac{2^{-N} \Gamma(1 / 2)}{\Gamma(N+M+1 / 2)} A_{M, N, K}
$$

и наложим условие $B_{N M K}=0$ при $N, M, K \leqslant 0, N \leqslant M \leqslant K$. Это позволяет получить соотношение

$$
\begin{aligned}
\delta_{M, 0} \delta_{N, 0} \delta_{K, 0}= & (4 N-2 M-2 K+1) B_{M N K}+B_{M, N-1, K-1}+B_{M, N-1, K}- \\
& -\hbar^{2}(N+M-1) B_{M-1, N-1, k} .
\end{aligned}
$$


Нетрудно вывести выражения замкнутого вида для некоторых наборов коэффициентов; например, справедливы формулы

$$
B_{N N 0}=\frac{\hbar^{2 N}}{1+2 N}, \quad B_{0 N 0}=\frac{(-1)^{N} \Gamma(5 / 4)}{4^{N} \Gamma(N+5 / 4)}, \quad N \geqslant 0 .
$$

Наконец, можно исключить зависимость от индекса $K$ из выражений для коэффициентов $B_{M, N, K}$ посредством преобразований. В самом деле, используя замену

$$
B_{M, N, K}=\left(\begin{array}{c}
2 N-M+1 / 2 \\
K
\end{array}\right) C_{M, N}
$$

мы приводим рекуррентное соотношение (94) к окончательному виду

$$
\delta_{M, 0} \delta_{N, 0}=(4 N-2 M+1) C_{M N}+C_{M, N-1}-\hbar^{2}(N+M-1) C_{M-1, N-1} .
$$

Это выражение в точности совпадает с рекуррентным соотношением для угловой переменной для квадратичного осциллятора [1].

В свете сказанного выше и результатов статьи [1] можно сформулировать следующее утверждение, чтобы обобщить основные свойства решения рассматриваемой задачи: минимальный оператор типа угол $\Theta_{0}$, сопряженный посредством уравнения $\left[H_{0}, \Theta_{0}\right]=i \hbar$ с гамильтонианом $H_{0}=P^{2}+Q^{4} / 2+Q^{2}$, имеет вид

$$
\Theta=\sum_{N=0}^{\infty} \sum_{M=0}^{N} \sum_{K=0}^{N-M}\left(\begin{array}{c}
2 N-M+1 / 2 \\
K
\end{array}\right) C_{M, N} \mathcal{T}_{-2 N-2 M-1,4 N-2 M-2 K+1},
$$

где коэффициенты $C_{m, n}$ можно определить выражением

$$
C_{M, N}=\left.\frac{1}{M ! N !}\left(\frac{\partial}{\partial_{x}}\right)^{M}\left(\frac{\partial}{\partial_{y}}\right)^{N} g(x, y)\right|_{x=y=0}
$$

в котором $g(x, y)$ есть производящая функция [1]

$$
g(x, y)=\int_{0}^{1} \frac{d \xi}{2 \sqrt{\xi}(1-x y \xi)} \exp \left\{\frac{1}{x^{2} y}\left(\frac{2}{3}-x y+\frac{(1-x y)^{3 / 2}(x y \xi-2 / 3)}{(1-x y \xi)^{3 / 2}}\right)\right\}
$$

В эквивалентной записи $(N+M \rightarrow R)$

$$
\begin{gathered}
\Theta=\sum_{N=0}^{\infty} \sum_{R=N}^{2 N} \sum_{K=0}^{2 N-R} \widetilde{C}_{N, R, K} \mathcal{T}_{-2 R-1,6 N-4 R-2 K+1} \\
\widetilde{C}_{N, R, K}=\left(\begin{array}{c}
3 N-R+1 / 2 \\
K
\end{array}\right) C_{R-N, N}
\end{gathered}
$$

В дальнейшем можно привлечь формулу (40), чтобы записать формальное решение $\Theta(q, p, t)$ операторного уравнения $[I, \Theta]=i \hbar$ с инвариантом $I$, заданным выражением (87). 


\section{8. ВЫВОДЫ}

В настоящей статье исследована проблема решения неавтономных операторных уравнений, связанных с коммутаторами в фазовом пространстве. Основной причиной, побудившей нас рассмотреть данный вопрос, явились попытки привнести основанную на симметрии структуру на квантовый уровень. Этот подход получил развитие после основополагающих работ [18], [19] и в настоящее время используется при рассмотрении понятия суперинтегрируемости (см., например, статьи [20]). По причине того, что подлежащие исследованию динамические объекты имеют различную природу и связанные с ними пространства имеют различные характеристики, для того чтобы в полной мере использовать структуру, основанную на симметрии, на квантовом уровне, необходимо понимание всех аспектов проблемы построения канонически сопряженных квантовых наблюдаемых и применения на квантовом уровне классических квантовых преобразований. Фактически в настоящей статье мы исследовали квантовые вопросы, близкие по смыслу проблеме интегрируемости по Лиувиллю в классической динамике, уделяя особое внимание представлению оператора, удовлетворяющему условию канонического спаривания, а также применению на квантовом уровне классических канонических преобразований.

Канонические преобразования, действию которых подвергается операторный базис в фазовом пространстве, являются линейными и зависят от времени. Это позволило сделать влияние зависимости от времени на реализацию базиса, получаемого после применения преобразований из класса, представляющих реальный интерес (см. работы [21]), особенно заметным. В настоящей статье была предложена точная формула, описывающая взаимосвязь двух канонических операторных базисов Бендера-Данна. Вместе с тем мы распространили некоторые результаты, уже полученные в этой области [11], на случай отрицательных степеней, в которые возводятся пространственные (соответственно импульсные) операторы, а именно, на случай отрицательных индексов $m$ (соответственно $n$ ) в формуле (12) для базисных элементов $T_{m, n}$, упорядочивая их стандартным способом и по Вейлю (см. выражения для $\mathcal{T}_{-m, n}^{\mathcal{C}}$ в п. 3.2).

Далее мы учли явную структуру угловых операторов, лежащих в основе неавтономных гамильтоновых систем, используя формальные операторные ряды в двух упомянутых выше базисах. Были рассмотрены неавтономные одномерные гамильтоновы системы, являющиеся линейными, квадратичными или системами четвертого порядка, и приведены подробные детали всех вычислений ${ }^{2)}$. Посредством применения минимальных операторов мы решили неизбежные проблемы, являющиеся следствием неединственности реализации операторов, удовлетворяющих коммутационным соотношениям. Были выписаны производящие функции для коэффициентов минимального [1] представления разложений решений операторных уравнений типа действие-угол для всех рассмотренных случаев.

При выборе согласованных с минималъными точных решений для операторов типа угол мы также установили связь с методом обратной задачи Лиувилля (см. приложение). Для зависящих от времени гамильтоновых систем полученные для коэффициентов минимальных искомых операторов типа угол рекуррентные соотношения позволяют в явном виде заново оценить операторную цепочку, через которую с использованием схемы обратной задачи Лиувилля формально выражаются

2) В дальнейшем возникают вопросы о сингулярном поведении операторов или возможности использования их положительных оперативных мер. 
решения для автономных систем. Было получено полное соответствие между минимальными решениями, найденными независимо в двух базисах, при сохранении в выражении для преобразованного базиса Бендера-Данна лишь вклада наименьшего порядка.

Для гамильтониана четвертого порядка минимальное решение формально не совпадает по структуре с решениями в классическом случае. Как было показано в разделе 4, данный факт был вполне ожидаем и может послужить поводом для дальнейших исследований. Они могут позволить получить ответ на вопрос о том, до какой степени на структуру решений, полученных в рамках классического предела, влияет включение в выражения для приближений операторов $\mathcal{T}_{m, n}$ первых не равных нулю членов, содержащих степени постоянной Планка. Фактически при высоких степенях эти члены перемешиваются с членами, отвечающими за отклонения от минимальных решений.

\section{Метод обратной задачи Лиувилля}

\section{ПРИЛОЖЕНИЕ}

Рассмотрим вкратце представленный в статье [2] формальный подход к исследованию операторного уравнения $[H, F]=i \hbar$ и его решений, где $H$ - гамильтониан системы. Лежащая в основе метода идея имеет следующий вид: если предположить, что существует оператор, обратный к оператору $\mathcal{L}_{H}=:[H, \cdot]$ (в случае аналитических потенциалов данная гипотеза верна), то можно записать решение как $F=i \hbar \hat{\mathcal{L}}_{H}^{-1}$. Преобразуем данное выражение. А именно, если выделить из гамильтониана кинетическую энергию $K$ и потенциальную энергию $V: H=K+V$, то оператор $F$, формально являющийся решением рассматриваемого операторного уравнения $[H, F]=i \hbar$ (или в форме Лиувилля $\mathcal{L}_{H} \cdot F=i \hbar 1$ ), дается геометрическим рядом $F=\sum_{k=0}^{\infty}(-1)^{k} F_{k}$, где через $F_{0}$ обозначен кинетический вклад, а все остальные члены можно получить с помощью рекуррентных соотношений

$$
F_{0}=\hat{\mathcal{L}}_{K}^{-1}(i \hbar), \quad F_{k}=\left(\hat{\mathcal{L}}_{K}^{-1} \hat{\mathcal{L}}_{V}\right) F_{k-1}, \quad k \geqslant 1
$$

Оператор $\mathcal{L}_{K}$, действующтий в пространстве псевдодифференциалов над $L^{2}(R)$, можно определить в терминах его действия на базисные операторы Бендера-Данна. Соответственно определяются и члены разложения.

Например, применение данного подхода в случае гамильтониана четвертой степени $H=p^{2}+q^{2}+q^{4}$ приводит к члену нулевой степени $F_{0}=-T_{-1,1} / 2$ и разложению

$$
F=-\frac{1}{2} \sum_{k=0}^{\infty}(-1)^{k}\left(\hat{\mathcal{L}}_{K}^{-1} \hat{\mathcal{L}}_{V}\right)^{k} T_{-1,1}
$$

а действие составного оператора $\hat{\mathcal{L}}_{K}^{-1} \hat{\mathcal{L}}_{V}$ на базис Бендера-Данна описывается выражением

$$
\begin{aligned}
\left(\hat{\mathcal{L}}_{K}^{-1} \hat{\mathcal{L}}_{V}\right) T_{-m, n}= & -2\left(\frac{m}{n+4}\right) T_{-m-2, n+4}+ \\
& +\frac{1}{2} \frac{m(m+1)(m+2)}{n+2} T_{-m-4, n+2}-\frac{m}{n+2} T_{-m-2, n+2} .
\end{aligned}
$$


Повторное применение формулы (П.3) в случае $m=n=1$ приводит к равенству

$$
\left(\hat{\mathcal{L}}_{K}^{-1} \hat{\mathcal{L}}_{V}\right)^{n} T_{-1,1}=\sum_{m=0}^{n} \sum_{k=0}^{m} A_{n, m, k} T_{-4 n+2 m-1,2 n+2 m-2 k+1}
$$

при некотором $n \geqslant 0$ и постоянных коэффициентах $A_{n, m, k}$. Легко показать по индукции, что равенство (П.4) верно при всех $n \geqslant 0$. В процессе доказательства также можно заметить, что рекуррентное соотношение, единственным образом определяющее неизвестные коэффициенты $A_{n, m, k}$ с граничным условием $A_{0,0,0}=1$, можно перегруппировать в виде, аналогичном выражению (90). Следовательно, минимальное решение совпадает с решением, полученным с помощью метода обратной задачи Лиувилля.

Благодарности. Д. Ландольфи благодарен The University of Tokyo, Institute of Industrial Science за гостеприимство.

\section{Список литературы}

[1] C. M. Bender, G. V. Dunne, "Exact solutions to operator differential equations", Phys. Rev. D, 40:8 (1989), 2739-2742; "Integration of operator differential equations", Phys. Rev. D, 40:10 (1989), 3504-3511.

[2] E. A. Galapon, A. Villanueva, "Quantum first time-of-arrival operators", J. Phys. A: Math. Theor., 41 (2008), 455302, 31 pp.

[3] H.-Y. Fan, H. R. Zaidi, J. R. Klauder, "New approach for calculating the normally ordered form of squeeze operators", Phys. Rev. D, 35 (1987), 1831; H.-Y. Fan, Y. Fan, "Representations of two-mode squeezing transformations", Phys. Rev. A, 54 (1996), 958.

[4] H.-Y. Fan, Y. Fan, "Newton-Leibniz integration for ket-bra operators in quantum mechanics and derivation of entangled state representation", Ann. Phys., 321:2 (2006), 480-494; H.-Y. Fan, "Newton-Leibniz integration for ket-bra operators in quantum mechanics (IV) - Integrations within Weyl ordered product of operators and their applications", 323:2 (2008), 500-526.

[5] C. M. Bender, M. Gianfreda, "Matrix representation of the time operator", J. Math. Phys., 53:6 (2012), 062102, 20 pp.

[6] C. M. Bender, M. Gianfreda, "Mariagiovanna Nonuniqueness of the $\mathscr{C}$ operator in $\mathscr{P} \mathscr{T}$-symmetric quantum mechanics", J. Phys. A: Math. Theor., 46:27 (2013), 275306, $18 \mathrm{pp}$.

[7] E. A. Galapon, F. Delgado, J. Gonzalo Muga, I. Egusquiza, "Transition from discrete to continuous time-of-arrival distribution for a quantum particle", Phys. Rev. A, 72:4 (2005), 042107, 8 pp.

[8] A. Mostafazadeh, "Time-dependent diffeomorphisms as quantum canonical transformations and the time-dependent harmonic oscillator", J. Phys. A: Math. Gen., 31 (1998), 6495.

[9] B. J. Leimkuhler, C. R. Sweet, "Hamiltonian formulation for recursive multiple thermostats in a common timescale", SIAM J. Appl. Dyn. Syst., 4:1 (2005), 187-216; S. Bond, B. Laird, B. Leimkuhler, "The Nosé-Poincaré method for constant temperature molecular dynamics. Computational molecular biophysics", J. Comput. Phys., 151 (1999), 114; S. Nosé, "An Improved symplectic integrator for Nosé-Poincaré thermostat", J. Phys. Soc. Japan, 70:1 (2001), 75-77.

[10] G. Landolfi, "Weyl-ordered series form for the angle variable of the time-dependent oscillator", J. Phys. A: Math. Gen., 41:18 (2008), 185302, 11 pp.

[11] M. A. Lohe, A. Thilagam, "Weyl-ordered polynomials in fractional-dimensional quantum mechanics", J. Phys. A: Math. Gen., 38:2 (2005), 461-483. 
[12] J. Struckmeier, C. Riedel, "Canonical transformations and exact invariants for timedependent Hamiltonian systems", Ann. Phys., 11:1 (2002), 15-38.

[13] M. Maamache, "Unitary transformation approach to the exact solution for a class of time-dependent nonlinear Hamiltonian systems", J. Math. Phys., 39:1 (1998), 161-169.

[14] A. Geralico, G. Landolfi, G. Ruggeri, G. Soliani, "Novel approach to the study of quantum effects in the early Universe", Phys. Rev. D, 69:4 (2004), 043504, 10 pp.

[15] C. M. Bender, G. V. Dunne, "Polynomials and operator orderings", J. Math. Phys., 29:8 (1988), 1727-1731.

[16] M. Gianfreda, G. Landolfi, "Spectral problems for the Weyl-ordered form of operators $\left(\frac{1}{p}\right)^{n} \hat{q}^{n} "$, J. Math. Phys., 52:12 (2011), 122104, 21 pp.

[17] J. Bunao, E. A. Galapon, "The Bender-Dunne basis operators as Hilbert space operators", J. Math. Phys., 55:2 (2014), 022102, 18 pp.

[18] A. O. Barut, A. Böhm, "Dynamical groups and mass formulas", Phys. Rev. B, 139:4 (1965), 1107-1112.

[19] Y. Dothan, M. Gell-Mann, Y. Ne'eman, "Series of hadron energy levels as representations of non-compact groups", Phys. Lett., 17 (1965), 148; Y. Dothan, "Finite-dimensional spectrum-generating algebras", Phys. Rev. D, 2 (1970), 2944-2954.

[20] P. Winternitz, I. Yurdusen, J. Math. Phys., 47:10 (2006), 103509, 10 pp.; W. Miller, Jr., S. Post, P. Winternitz, J. Phys. A: Math. Theor., 46:42 (2013), 423001, 97 pp.

[21] Y.S. Kim, E. P. Wigner, "Canonical transformation in quantum mechanics", Amer. J. Phys, 58:5 (1990), 449-451; A. J. Bordner, "Linear canonical transformations in quantum mechanics", J. Math. Phys., 38:7 (1997), 3427-3434; K.-H. Yeon, D. F. Walls, C. I. Um, T. F. George, L. N. Pandey, "Quantum correspondence for linear canonical transformations on general Hamiltonian systems", Phys. Rev. A, 58:3 (1998), 1765-1774; T. Hakiog̃lu, "Linear canonical transformations and quantum phase: a unified canonical and algebraic approach", J. Phys. A: Math. Gen., 32:22 (1999), 4111-4130.

Поступила в редакцию 26.07.2016, после доработки 18.11.2016 